\title{
Acute myocarditis and its consequences in Sweden
}

\author{
E. BengtSSON
}

THIS contribution concerns the clinical challenge of acute post-infectious myocarditis mainly from two aspects: the signs and symptoms in the acute stage based on material of mixed common infections, and the prognosis as based on a 5 year follow-up study. Regarding the latter group, acute myocarditis might be considered in the pathogenesis of cardiomyopathy.

\section{Acute myocarditis}

The frequency of acute myocarditis varies with age and aetiology, being twice as common in adults as in children, with the exception, however, of Coxsackie carditis in infants. It has a rate of about $5 \%$ in both streptococcal and Coxsackie infections and is also common in poliomyelitis and mumps. The frequency also varies with the severity of the underlying disease. ECG changes were found in $5-10 \%$ in non-paralytic and in $30 \%$ of paralytic poliomyelitis patients; in fatal bulbar paralytic cases all had histological evidence of acute myocarditis (Nordenstam, 1956). In mumps there was a higher frequency in patients with concomitant meningitis. On the other hand signs of myocarditis are scanty in some other diseases such as whooping cough and measles (at least in children) and extremely rare in viral hepatitis. The onset of myocarditis was traced histologically to the viraemic prodromal stage in patients with severe poliomyelitis dying within $24 \mathrm{hr}$ of admission to hospital. It is, however, delayed to several weeks after onset of some diseases caused by toxins and protozoa as well as in some other viral diseases.

\section{Clinical presentation}

A few patients develop overt signs of right or left heart failure or both; in most patients, however, signs and symptoms are subclinical. The response to exercise tests demonstrates lack of cardiac reserve, diminished increase of cardiac output or pulmonary arterial pressure, as well as decrease in the peripheral pressure. The findings indicate that the normal relaxation and filling of the ventricles are restricted and that the normal contraction and ejection powers are impaired. Thus, the clinical findings can chiefly be ascribed to impaired ventricular contractibility and dilatation (Bengtsson, 1957b; Gotsman et al., 1971). To this are added the common disturbances in rhythm and conduction.
Clinically, the most common signs are relative tachycardia, hypotension, orthopnoea, triple rhythm, arrhythmia, precordial pains and, in children and infants, liver enlargement and cyanosis. Very often, however, signs and symptoms are scanty and equivocal. In these cases various function tests are of interest. We use the bicycle ergometer test, measuring the submaximal working capacity at a pulse rate of $170 / \mathrm{min}$ and correlating it to the heart volume (the parameters corresponding to decreased stroke volume and ventricular dilatation respectively). Exercise ECG, examined simultaneously with the bicycle ergometer test was of decisive diagnostic importance in relation to resting ECG in a third of the patients.

Signs and symptoms of cardiac insufficiency seem to persist in many patients. Out of a total of about 15,000 patients with acute infectious diseases from the early 1950s about 200 were accepted in whom extracardiac factors and diseases other than myocarditis could be excluded (Bengtsson, 1957a). This series was compared with a series of patients with dubious signs of myocarditis and a group without any signs of myocarditis. Most of them were reexamined 5 years afterwards using the same methods (Bengtsson \& Lamberger, 1966).

At that time $20 \%$ in the first group (definite myocarditis) still had subjective symptoms, tiredness, shortness of breath on effort, palpitations and/or precordial discomfort. Systolic murmurs were found in patients who had had streptococcal infections 5 years earlier and occasionally in patients with other diseases, even in viral infections. Among electrocardiographic abnormalities rhythm disturbances were found mainly in the non-streptococcal group, predominantly in the viral myocarditis patients. Conduction disturbances on the other hand were found only among the rheumatic patients. Changes in the terminal complex were more evenly distributed.

Abnormal heart volumes were found as often in the rheumatic as among the aseptic meningitis group. Nor was there any difference in the ergometer tests between the rheumatic and non-rheumatic group the working capacity being impaired in about $40 \%$. However, only occasional patients were handicapped in their work. Some patients might have contracted new acute myocarditis during the previous 5 years. 
TABLE 1. Incidence of signs and symptoms in three groups first examined 5 years earlier: patients without signs of cardiac involvement (controls), patients with dubious myocarditis, and patients with presumptive myocarditis (Bengtsson \& Lamberger, 1966)

\begin{tabular}{|c|c|c|c|c|c|c|}
\hline \multirow[t]{2}{*}{ Follow-up findings } & \multicolumn{2}{|c|}{$\begin{array}{c}\text { Controls } \\
\text { (34) }\end{array}$} & \multicolumn{2}{|c|}{$\begin{array}{l}\text { Dubious } \\
\text { myocarditis } \\
(26)\end{array}$} & \multicolumn{2}{|c|}{$\begin{array}{l}\text { Presumptive } \\
\text { myocarditis } \\
(90)\end{array}$} \\
\hline & Number & $\%$ & Number & $\%$ & Number & $\%$ \\
\hline Subjective symptoms & 2 & & 7 & 27 & 22 & 24 \\
\hline \multicolumn{7}{|l|}{ Elevated antistreptolysin } \\
\hline titer and/or ESR & 8 & 23 & 4 & 15 & 25 & 28 \\
\hline Abnormal PCG findings & 1 & & 5 & 19 & 21 & 23 \\
\hline Abnormal resting ECG & 0 & & 3 & 12 & 17 & 19 \\
\hline Abnormal orthostatic ECG & 4 & 12 & 7 & 27 & 9 & 10 \\
\hline Abnormal exercise ECG & 2 & & 7 & 27 & 27 & 30 \\
\hline (Total with ECG changes) & 5 & 14 & 13 & 50 & 44 & 49 \\
\hline Low working capacity & 1 & & 12 & 46 & 34 & 41 \\
\hline Enlarged heart volume & 0 & & 5 & 19 & 17 & 19 \\
\hline
\end{tabular}

PCG, phonocardiogram.

Judging from the results of the control group this attack rate might have been approximately $10 \%$. Thus, the overall result of the study indicates that about $30 \%$ of the patients 5 years after acute myocarditis still had signs of cardiac insufficiency, almost all sub-clinical. This figure from mixed material where rheumatic fever was included is almost the same as the figure from South Africa (Sainani, Krompotica \& Slodki, 1968) from a series of twenty-two patients with true Coxsackie infections, and is also similar to other series from Sweden (Bengtsson et al., 1970; Levander-Lindgren, 1965).

During the 5-year period of observation I found no patient with definitive signs of progressive heart hypertrophy or dilatation. On the other hand there must be a great many people who contract undiagnosed acute myocarditis and many years afterwards have palpitations and shortness of breath and demonstrate slight increases of heart volume and abnormalities in resting or exercise ECG. It is a question of definition if they should be included among the 'cardiomyopathies'.

\section{References}

BENGTSSON, E. (1957a) Electrocardiographic studies in patients with abnormalities in serial examinations with standard leads during acute infectious diseases. Acta medica scandinavica, 159, 395.

BENGTSSON, E. (1957b) Working capacity and heart volume in patients with electrocardiographic abnormalities suggestive of acute myocarditis during various acute infectious diseases. Acta medica scandinavica, 159, 499.

Bengtsson, E. \& Lamberger, B. (1966) Five-year follow-up study of cases suggestive of acute myocarditis. American Heart Journal, 72, 751.

Bergström, K., Erikson, U., Nordbring, F., Nordgren, B. \& PARRow, A (1970) Acute non-rheumatic myopericarditis: a follow-up study. Scandinavian Journal of Infectious Diseases, $2,7$.

Gotsman, M.S., van DER Horst, L., Winship, W.S. \& SCHAEFFER, T.G. (1971) Heart size in primary myocardial disease. South African Medical Journal, 45, 744.

LEVANDER-LINDGREN, M. (1965) Studies in myocarditis. III. On the frequency and nature of some common symptoms. Cardiologia, 47, 139.

Nordenstam, H. (1956) Pathoanatomical findings in twentysix fatal cases of poliomyelitis. Acta medica scandinavica, Suppl. 316, 114.

Sainani, G.S., Krompotic, E. \& Slodki, S. (1968) Adult heart disease due to the Coxsackie virus $B$ infection. Medicine, 47, 133. 